Background Failure to properly dispose of self-DNA can inappropriately trigger anti-viral defense systems, leading to autoimmunity. Indeed, mutations in the DNA exonuclease TREX1 are causative for a spectrum of rare lupus-like autoimmune diseases in humans. These disorders involve triggering of the cytosolic dsDNA sensor cyclic GMP-AMP Synthase (cGAS) and the STimulator of INterferon Genes (STING), leading to chronic production of the anti-viral cytokine type I interferon (IFN-I) and the development of autoimmunity. Importantly, the exact cells in which the sensing of undegraded DNA and subsequent production of IFN-I occur remain unknown.

Methods We generated a mouse expressing the catalytically inactive TREX1 D18N allele, which causes familial chilblain lupus in humans. We examined anti-viral gene expression and the phenotype of these mice to study the immunological effects of losing TREX1 activity. We performed bone marrow transplants to determine if autoimmune pathogenesis in this model was dependent on hematopoietic or nonhematopoietic cells. Finally, we measured expression of type I interferon is various purified cell populations to identify specific cellular producers contributing to autoimmune pathogenesis.

Results In this study, we demonstrate that TREX1 catalytic inactivity induces IFN-I signaling and lupus-like autoimmunity in a mouse. Moreover, we show that TREX1 deficiency within bone marrow-derived cells causes IFN-I activation and the development of autoimmunity. We provide evidence of spontaneous IFN- production within both innate immune and $\mathrm{T}$ cells. $\mathrm{T}$ cell IFN-a expression was observed in all $\mathrm{T}$ cell populations, but was most enriched within naive $\mathrm{T}$ cells. We also demonstrate that $\mathrm{D} 18 \mathrm{~N}$ T cells express all components of the cGAS-STING pathways and generate IFN-I protein, both spontaneously and in response to small-molecule activation of STING.

Conclusions Our findings demonstrate that TREX1 enzymatic activity is crucial to prevent inappropriate DNA-sensing and IFN-I production. TREX1 inactivity within hematopoietic cells was both necessary and sufficient to induce lupus-like autoimmunity, indicating that TREX1 normally acts within immune cells to suppress inappropriate activation of anti-viral signaling. Both innate immune and $\mathrm{T}$ cells respond to TREX1 dysfunction by spontaneously synthesizing IFN-, a surprising result given that $\mathrm{T}$ cells are not canonically thought to be major IFN-producing cells. These results expand our understanding of the pathogenesis of lupus-like disease, and indicate that small molecule inhibition of TREX1 could represent an appealing strategy for anti-viral and cancer immune-therapies.

Funding Source(s): NIH grant - 5R01AI116725-03

$\mathrm{NIH}$ training grant - 5T32AI007401-23

\section{LUPUS NEPHRITIS PRESENTING AS TUBERCULOSIS INFECTION: A CASE REPORT}

Muhammad T Khan*. Dow University Hospital

\subsection{6/lupus-2019-Ism.285}

Background Lupus nephritis, a form of glomerulonephritis, is the inflammation of kidneys attributable to systemic lupus erythematosus (SLE). It is an autoimmune disorder in which kidney tissue insult is triggered predominantly by the complement system. Herein, we report the case of a 24 years old female with atypical manifestations of SLE.

Methods Case Presentation: A female aged 24 years was presented with the complaint of fever, cough (with sputum) and dyspnea for the past one month. The preliminary physical examination was suggestive of pneumonia. Her laboratory investigations revealed anisocytosis and normochromic anemia ( $\mathrm{Hb} 9.3 \mathrm{~g} / \mathrm{dL}$, HCT 30\%). Lymphocytes (12\%) were decreased while neutrophils $(84 \%)$, platelets $(439 \times 103 / \mu \mathrm{l})$ and ESR $(75 \mathrm{~mm} / \mathrm{hr})$ were increased. A serum biochemistry test showed elevated urea $(92 \mathrm{mg} / \mathrm{dl})$, creatinine $(1.5 \mathrm{mg} / \mathrm{dl})$ and sodium $(145 \mathrm{meq} / \mathrm{L})$. The chest X-ray demonstrated a right sided pleural effusion which directed towards a possible Tuberculosis (TB) infection. However, pleural biopsy efficiently excluded an active TB infection. Nephrological investigations exhibited evidence of protein $(0.3 \mathrm{~g} / \mathrm{L})$ and blood traces in the urine. Her proteinuria $(2+)$ was within nephrotic range which was confirmed through qPCR (3.9 U). Moreover, serum TSH $(8.054 \mathrm{mU} / \mathrm{L})$ was also elevated and cardiolipin test was positive for IgM (1.10). SLE specific diagnostic tests anti-dsDNA was positive and ANA was also reactive. Left kidney biopsy exhibited characteristics of diffuse endocapillary proliferative glomerulonephritis. SLE diagnosis was established, and patient was treated with cyclophosphamide pulse therapy along with corticosteroid methylprednisolone and achieved complete remission.

Results CASE REPORT

Conclusions To date, this is the first case report of SLE simulating as a TB infection in a developing country. The patient did not display classic triad of SLE; joint pain and malar rash aside from fever. This case reiterates the implication of considering unusual case presentations of SLE and undertaking rigorous clinical workup to minimize the probability of missed cases and improve patient clinical outcomes.

Funding Source(s): None

\section{\begin{tabular}{|l|l}
286 & MATERNAL SYSTEMIC LUPUS ERYTHEMATOSUS AND
\end{tabular} THE RISK OF CRYPTORCHIDISM IN MALE OFFSPRING}

${ }^{1}$ Signe Schollhammer Knudsen*, ${ }^{2}$ Bodil Hammer Bech, ${ }^{3}$ Bent Winding Deleuran, ${ }^{2}$ Cecilia Høst Ramlau-Hansen, ${ }^{2}$ Linn Håkonsen Arendt. 'Stanford University School of Medicine; ${ }^{2}$ Aarhus University; ${ }^{3}$ Aarhus University, Aarhus University Hospital

\subsection{6/lupus-2019-Ism.286}

Background Background: Systemic lupus erythematosus (SLE) is one of the most prevalent autoimmune rheumatic diseases affecting young women of childbearing age. The disease is characterized by elevated levels of systemic inflammation and high levels of circulating autoantibodies, potentially affecting multiple organs and tissues. Women suffering from SLE have decreased fecundity, and increased risk of adverse pregnancy outcomes such as miscarriage and preeclampsia. This risk is further elevated with increasing disease activity, and seems less pronounced when disease is well managed. SLE is not in itself considered a risk factor for specific birth defects; however, not all congenital anomalies are diagnosed immediately after birth, and might thus be overlooked in most studies on reproductive outcomes. Cryptorchidism (undescended testis) is a common genital anomaly, often diagnosed throughout childhood. It arises from an imperfect genital development in the 
foetus. As the prevalence of cryptorchidism in male offspring is higher when their mothers are suffering from preeclampsia or gestational diabetes, we aimed to investigate if a similar association exists between maternal SLE and cryptorchidism in the offspring.

Methods Methods: We conducted a nationwide study including all male singleton live births in Denmark from 1995 to 2016. Using the Danish nationwide population based registers, we assessed the occurrence of cryptorchidism according to prenatal disease-state of the mothers (SLE/no SLE). Cryptorchidism was assessed as both any diagnosis of cryptorchidism and diagnosis of cryptorchidism and corrective surgery. Using Cox proportional hazards models we calculated hazard ratios (HRs), accounting for varying age at time of diagnosis, and adjusting for maternal age, smoking during pregnancy, pre-gestational BMI, parity, educational level and ethnicity.

Results Among 690240 boys, 352 boys were born of mothers with pre-existing SLE. Of those, 15 (4.3\%) boys were diagnosed with cryptorchidism, and seven boys (1.9\%) underwent corrective surgery. We found an adjusted HR of 1.68 (95\% CI: 1.01, 2.78) for cryptorchidism and 1.46 (95\% CI: $0.69,3.06)$ for cryptorchidism with corrective surgery, among boys born by mothers with SLE, compared with unexposed boys. This is consistent with an increased risk of cryptorchidism among the exposed boys, even though the relatively few exposed cases limits the precision of the estimates.

Conclusions Conclusions: Boys exposed to maternal SLE appears to have higher risk of cryptorchidism, compared with unexposed boys.

Funding Source(s): Aarhus University, the Danish rheumatism society and Karen Elise Jensen foundation.

\section{7 AFRICAN AMERICAN SLE PATIENTS WITH VARIABLE DISEASE ACTIVITY REVEAL ALTERATIONS IN SIGNALING PATHWAYS AND SOLUBLE MEDIATORS THAT ARE MORE PRONOUNCED THAN EUROPEAN AMERICAN PATIENTS}

${ }^{1}$ Samantha Slight-Webb, ${ }^{1}$ Miles Smith, ${ }^{2}$ Eliza Chakravarty, ${ }^{1}$ Cristina Arriens, ${ }^{1}$ Teresa Aberle,

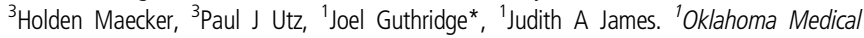
Research Foundation; ${ }^{2}$ Oklahoma Medical Research Foundation; University of Oklahoma; ${ }^{3}$ Stanford University

\subsection{6/lupus-2019-Ism.287}

Background Systemic lupus erythematosus (SLE) is an autoimmune disorder with a variable clinical presentation and periods of waxing and waning disease. Heterogeneity in SLE is influenced by genetic and non-genetic susceptibility found in different ethnicities that drive disease expression and severity. The immune pathways that contribute to heightened disease activity in lupus and immune variation by race are critical to understanding SLE disease mechanisms and outcomes.

Methods Peripheral whole blood samples of European or African American healthy controls $(\mathrm{n}=18)$ and SLE patients with either high (SLEDAI4) $(n=20)$ or low (SLEDAI $<4)(n=20)$ disease activity were stimulated for 4 min with either interferon-(IFN), PMA and ionomycin, or Toll-like receptor (TLR) ligands for either TLR4, TLR7/8 or TLR9 for phospho-protein analysis, or 24 hours for cytokine analysis of cell culture supernatants. Phenotype and phospho-protein analysis was assessed by CyTOF and cell heterogeneity was analyzed using t-SNE and manual gating. Soluble mediators were assessed using 37-plex xMAP assays and ELISA. All SLE patients met ACR classification criteria.

Abstract 287 Figure 1 African Americans SLE patients with high disease activity exhibit greater dysregulation in phospho-signaling following stimulation. Peripheral whole blood from either European or African American healthy controls, SLE patients with low disease activity (SLEDAl<4) and SLE patients with high disease activity (SLEDAI $\geq 4$ ) were stimulated for 4 minutes with either no stimuli, IFN $\alpha$, PMA and ionomycin, or TLR4, TLR7/8 or TLR9 agonists. Data was collected via mass cytometry, and analyzed using Cytobank. The median 95th percentile wase used to calculate the fold change of high disease activity patients over healthy control of low disease activity patients in African American (left) or European Americans (right) in 8 cell population (B cells, CD4+ T cells, CD8+ T cells, dendritic cells (DCs), plasmacytoid DCs (pDCs), natural killer (NK) cells, monocytes (mono) and granulocytes (gran). Significant increases or decreases in phophorylation following stimulation in a particular signaling molecule are noted by a green box (decrease) or a red box (increase). The location of the dot coincides with significant differences found in a cell population (refer to legend). $p<0.05$ 